\title{
Disk-dispersal and planet-formation timescales
}

\author{
Lynne A Hillenbrand \\ California Institute of Technology; Mail Code 105-24; Pasadena CA 91125, USA \\ E-mail: 1ah@astro.caltech.edu
}

Received 11 March 2008

Accepted for publication 14 March 2008

Published 16 July 2008

Online at stacks.iop.org/PhysScr/T130/014024

\begin{abstract}
Well before the existence of exo-solar systems was confirmed, it was accepted knowledge that most - if not all — stars possess circumstellar material during the first one-to-several million years of their pre-main sequence lives, and thus that these systems commonly have the potential to form planets. Here, I summarize current understanding regarding the evolution of protoplanetary disks.
\end{abstract}

PACS numbers: 97.82.-j, 97.82.Fs

\section{Introduction}

From the analysis of the chemical record as traced through meteoritic material, we can infer the detailed history of the formation and early evolution of our planetary system (e.g. Foing 2008). Since the discovery of the first exosolar planet more than a decade ago, it has been understood that planetary systems around other stars could have properties very different from those characterizing the familiar solar system. Further, the biases and systematics that are inherent in the many exosolar planet detection techniques mean that the discovery of planetary systems resembling our own-in detail-remains for the future.

The great diversity in the measured orbital and internal properties among known exoplanets suggests that it would not be surprising were there similar diversity in the properties of the circumstellar disks out of which such planets form. Indeed, there is a wide range in inferred disk size, disk mass, disk geometry/structure and disk composition/chemistry, as touched upon by other authors in this volume (e.g. Henning 2008, Natta 2008, Dullemond et al 2008 and Aikawa and Namura 2008). While the initial conditions for planet formation do appear diverse, typical early-stage properties are: $M_{\text {disk }}=0.005 M_{\odot}$ (Osterloh and Beckwith 1995, Andrews and Williams $2005, \quad 2007) ; \quad M_{\text {disk }} / M_{\text {star }}=1-10 \% ; \quad \mathrm{d} M_{\text {acc }} / \mathrm{d} t=$ $10^{-8} M_{\odot} \mathrm{yr}^{-1}$ (Gullbring et al 1998, Muzerolle et al 2003, White and Basri 2003) and $R_{\text {disk }}=10-100$ AU (Dutrey et al 1996). We note that the dispersion can be orders of magnitude for some disk properties.

How does gas and dust that are initially well mixed and smoothly varying with radius and height turn into dynamically and compositionally diverse planetary systems? Here, we discuss the process of disk dissipation and presumed planet building, with particular attention to the current observational constraints on relevant timescales. We focus on stellar populations as a whole, rather than describing the many individual cases of disk evolution 'in action,' e.g. lines of evidence for grain growth which are covered by other authors in this volume. Theory has advanced in recent years to the point of making specific predictions for the evolution of quantities that, in principle, can be observationally constrained: surface density with radius (dissipation is inside/out in some models); total disk mass (nearly constant in some models); disk accretion (ceases entirely in some models); gas-to-dust ratio (decreases from $\sim 100$ to $<1 \%$ ); grain size distribution (increasing mean grain size); and chemical composition (set by response to x-ray, EUV, FUV and optical photon heating from the star). While some of these measures are still beyond the realm of observational constraint, other tests having statistical significance are possible now. A potential 'second parameter' effect is the local environment of the young star/disk system, and whether it is dynamically or radiatively important to the disk evolution (see Adams 2008 for discussion of cluster effects and Monin et al (2007) for a review of stellar and substellar companion properties and their effects).

\section{Planet building}

Observational probes of the planet-formation process are directed toward understanding the evolution of circumstellar dust gas properties. The basic processes on the dust side are 
those of decoupling from the gas, drift and mid-plane settling, coagulation and growth into larger grains, and consequent growth to macroscopic bodies termed 'planetesimals' which can become 'oligarchs' and eventually terrestrial-sized planets. These processes may be traced by studying, e.g. opacity versus wavelength, grain size, total disk mass, structure, etc. On the gas side, there is chemical evolution and final accretion of gas - if giant planets are formed - onto massive cores that were accreted themselves via the dust evolution processes just described. Tracers of gas disk evolution include probes of accretion onto the star as well as detection of gaseous species in more quiescent regions of the disk.

These planet-building processes all occur against the backdrop of viscous disk evolution (Alexander and Armitage 2007, Hartmann et al 1998, Takeuchi et al 2005), outflow via stellar/disk winds (Pudritz et al 2007), ionization/ photoevaporation (Gorti and Hollenbach 2008, Hollenbach et al 1994) and radiative blowout of small grains once the disk becomes optically thin. Various timescales are involved, ranging from those relevant to radiative transfer and chemistry to those which scale with the dynamical time in the disk. Here, we discuss mostly the dust disk evolution, since observational constraints on gas disk evolution are more limited at present. Both remain hindered to some extent by observational sensitivity, despite significant advancement in recent years.

How long does disk dissipation take, whether due to planetary formation or other processes? It has been known for some time (e.g. Nordh et al 1996 with ISO, Skrutskie et al 1990 working with IRAS) and confirmed with more recent data (e.g. Furlan et al 2006 and Hartmann et al 2005 with Spitzer) that the mid-infrared $(3-25 \mu \mathrm{m})$ colors and SED slopes of young low-mass stars (also known as T Tauri stars) in a single cluster segregate into two groups. They are interpreted as the stars with disks and the stars without disks. As the wavelength considered is decreased, blurring between the excess/disked and the non-excess/non-disked samples increases. That there are no or very few objects of intermediate color or spectral slope found at mid-infrared and longer wavelengths among stellar populations of nominally the same age, has been used to argue (e.g. Simon and Prato 1995, Wolk and Walter 1996) that the transition time from optically thick to optically thin disks is only a few hundred thousand years, i.e. that the disk-dissipation process is quite rapid-once it starts. Bertout et al (2007) and Hartigan et al (1995), however, the present evidence contrary to the so-called 'CTTS' and 'WTTS', i.e. Classical accreting and weak-/non-accreting T Tauri stars, are distinguishable in their luminosity (sic age) distributions.

The key to understanding whether the apparent diversity in the disk-dissipation timescale is reflective of differences in the duration of the process, or of those in the initial conditions within which the process occurs, may instead be related to its initial state or 'when it starts'. Similar bifurcation in mid-infrared colors is seen not only among very young $1-2 \mathrm{Myr}$ populations, but also in somewhat older 5-10 Myr populations, such as the $\eta$ Cha (Megeath et al 2005) and TW Hya (Low et al 2005) small associations. Noteworthy is that self-similar, viscous disk-evolution models (e.g. Hartmann et al 1998) do not yield this rapid transition between strong and weak/no disks that is observed. Models which invoke inside-out ionization/photoevaporation (e.g. Alexander and Armitage 2007) or rapid grain growth and planetesimal formation (e.g. Bauer et al 2008) may.

How long will known disks last? One relevant experiment is to consider the well-studied young stellar population of the Taurus-Auriga region for which (1) detailed spectral-energy distributions including data from the Spitzer Space Telescope; (2) disk masses from millimeter measurements and (3) mass accretion rates from inner disk to star measured via high-dispersion spectroscopic measurements of 'veiling' or lower dispersion direct detection of the Balmer continuum, are all available. A simple division of $M_{\text {star }} /\left(\mathrm{d} M_{\text {acc }} / \mathrm{d} t\right)$ yields a timescale - ranging from a few Myr to roughly a Gyr-for the accretion of material on to the central star. That these times are much longer, in the mean, than the inferred stellar ages indicates that the accretion rates must have been much higher in the past in order to build up the stellar mass to its present value. This point has been made before in the literature, repeatedly.

The complementary division of $M_{\mathrm{dust}} /\left(\mathrm{d} M_{\mathrm{acc}} / \mathrm{d} t\right)$ also yields a timescale, that for the disk to dissipate under the assumption that all of the material currently residing in the disk eventually winds up on the star. These inferred times are factors of several longer-than-the-current, presumed stellar ages of 1-2 Myr, suggesting that the disks will last well into the future. Given the simplistic assumptions regarding dust opacities used in estimating dust masses, they are likely underestimated, perhaps by an order of magnitude (e.g. Draine, 2006). This would strengthen our argument regarding the potential for 'long-lived' dust disks. When considered relative to typical theoretical timescales for planetary core formation and gas accretion, all young disks with substantial dust and gas thus appear to have the potential to form planets and can be considered proto-planetary.

\section{Transitional disks}

A category of disks which has been identified for close to 20 years but has only recently become well defined, is the so-called 'transition' disk sample. These objects make up a very small fraction of the total disk population. They are characterized as those disks having low dust content in the inner $(<1-10 \mathrm{AU})$ regions based on no or weak nearand mid-infrared excesses, perhaps indicative of an 'evolved' nature, yet they appear 'unevolved,' with strong excess emission and quite diverse spectral-energy distributions, at longer mid-infrared (e.g. Furlan et al 2006, Watson et al 2007) through sub-millimeter(mm)/mm wavelengths. They have a range of inferred dust masses that is typical of the disked T Tauri population. The 'transitional' disks are found to have low inner-disk gas content, where measurements are available, with gas surface densities $<1-2 \mathrm{~g} \mathrm{~cm}^{-2}$ and gas : dust ratios 250-1000 inferred at 0.5-1 AU (Herczeg et al 2007, Saylk et al 2007). This is consistent with the much lower-than-average accretion rates inferred from optical and ultraviolet diagnostics. According to the $M_{\mathrm{dust}} /\left(\mathrm{d} M_{\mathrm{acc}} / \mathrm{d} t\right)$ metric applied above, the 'transition' disk sources are 
projected to be exceptionally long-lived accretion systems, if their future evolution is determined entirely by viscous dissipation. However, dust/gas removal may occur by other means, for example, enhanced photoevaporation given that the inner disk is already cleared and the outer disk thus more directly illuminated by stellar photons.

The list of candidate 'transition' disks is growing rapidly based on sensitive Spitzer data. In well-studied star-forming regions, Spitzer has confirmed that many objects with only upper limits to their mid-infrared fluxes measured by IRAS, indeed have photospheric spectral-energy distributions with an upturn at wavelengths longer than 5-20 $\mu \mathrm{m}$. In other less well-studied regions, Spitzer by itself has characterized the entire strong/transitional/weak or non-disk population (e.g. Sicilia-Aguilar et al 2006a, 2006b).

The relative paucity of 'transitional' objects has long been used to argue that the phase from optically thick accretion to optically thin dust- and gas-poor disks is short-lived. One interesting question to ask is what are the ages of the known transitional systems, and how do they compare with those of the typical cluster or group member? We find that there is no difference in the mean, median, or distribution of (via K-S test) ages for the two populations in Taurus-Auriga. This finding is related to the well-appreciated dilemma of CTTS and WTTS mixing in the Hertzsprung-Russell diagram (HRD), noted earlier.

It is thus the case that stars of apparently the same age can have very different disks. This diversity is noted from consideration of bulk disk properties (presence/absence, total mass, size, accretion rate, etc) as well as in the many details of dust grain size and composition that are available from surface layer spectroscopy or thermal emission from near the mid-plane (see e.g., Natta 2008).

\section{Stellar ages}

This brings us to the discussion of stellar ages. While there are a number of different empirically calibrated stellar age estimation techniques for stars between $\sim 100 \mathrm{Myr}$ and $\sim 10$ Gyr old, for young stars, we are essentially stuck with the HRD as the only tool to determine stellar ages. I offer some cautionary words about what we can and what we should not believe about stellar ages inferred from individual measurements of $\log L / L_{\odot}$ and $\log T_{\text {eff }}$ for young stars.

Firstly, there are systematic concerns. For example, several $5-10 \%$ precise values of the distance to the Orion Nebula Cluster have been available very recently (Hirota et al 2007, Jeffries 2007, Menten et al 2008, Sandstrom et al 2007), indicating that all previous interpretations of the HRD for this cluster have suffered from systematic overestimate of the stellar luminosities and hence underestimate of the stellar ages. Another systematic effect is that of unresolved binarity, the implications of which on stellar luminosity estimates remains poorly characterized even for very well-studied clusters.

A further concern is that pre-main sequence evolutionary tracks vary substantially and systematically between theory groups. A comparison between those available reveals a trend in inferred stellar age that is relatively flat (i.e. consistent between the various groups producing the evolutionary tracks) for earlier type $F$ and $G$ stars, but increasing to about 0.75 dex (i.e. systematically and strongly varying between theory groups) for later type $\mathrm{K}$ and $\mathrm{M}$ young stars. In addition to the model-to-model systematics, it seems that all currently available sets of tracks under-predict stellar masses by 30-50\% (Hillenbrand and White 2004), while simultaneously under-predicting low mass stellar ages by $30-100 \%$ and over-predicting high mass stellar ages by $20-100 \%$, (Hillenbrand et al 2008). Finally, from comparison of presumably co-eval populations in pre-main sequence clusters, it is concluded that the higher mass stars systematically appear older than the lower mass stars in the same cluster, regardless of adopted tracks or mean cluster age. Although observers are generally grateful to have the opportunity to impose theoretical interpretation on their data, the above findings suggest that detailed work in stellar astrophysical theory is still needed, as is guidance from dynamical mass measurements across the pre-main sequence.

Secondly, there are random errors having to do with the accuracy of observationally determined quantities. These errors act to broaden luminosity dispersion, which is often (quite erroneously) interpreted as evidence for true age dispersion. Sources of random error include both astrophysical noises such as photometric variability, stellar/disk 'activity' and observational noise such as pure Poisson error in the measurements along with specific conditions, such as source crowding or high background, and other non-photospheric emission levels.

How much confidence can we place in stellar ages and hence inferred evolution of other physical variables based upon them? At present, a conservative estimate is that ages are accurate to factors of not better than $\sim 3$, including both systematic and random uncertainties. Prudence thus dictates caution regarding the inference of, e.g. star-formation histories in molecular clouds and cloud-free stellar associations, as well as in assessment of evolutionary timescales for e.g. circumstellar disks or stellar angular momentum.

A separate question beyond absolute age calibration, is whether all stars in a particular stellar cluster or association have the same age, or if there is evidence for age dispersion among cluster members (e.g. Tout et al 1999). We have been running Monte Carlo simulations to test whether observed luminosity distributions are consistent with error distributions or, perhaps, indicative of true age spreads (e.g. Hillenbrand et al 2007). The evidence at this point suggests that vast majority of cluster stars are consistent within the errors having the same age, i.e. there are no discernable age spreads representing the bulk of young stellar populations. Admittedly, there are some stars out on the tails of the luminosity distributions that seem hard to explain unless they are individually suffering some large unidentified source of error. St34 in Taurus, which-appears on the old side, or PDS66 in Upper Sco, which appears on the young side, are examples of such objects which as a class remain enigmatic at present.

\section{Current understanding of disk dissipation}

Returning now to the question of the timescale for disk dispersal and planet formation, we proceed by assembling 
(1) a set of stars which are known members of young associations or clusters, (2) the optical and/or near-infrared information which allows us to locate them in the theoretical HR diagram and (3) a quantitative disk diagnostic. Associations and clusters are useful bins because they provide statistical robustness, offer a sample over a range of stellar and substellar masses and provide stars assumed to have the same formation environment, metallicity and stellar age (probably, but see above). The optical and near-infrared photometric/spectroscopic data assembly is fairly 'bread-and-butter' at this point in astronomical history, requiring only time/effort and a careful assessment of errors. For the last need of a disk disagnostic, we could choose an optical depth indicator, such as monochromatic infrared excess at one or more wavelengths, a measure of total disk mass or a measure of the disk-accretion rate. For reasons of relative abundance and uniformity, we opt to make use of the infrared excess as our diagnostic.

Dust grains radiate over a broad range of wavelengths depending on their temperature and size. We can measure excess infrared emission due to absorbing and thermally re-emitting dust in the circumstellar environment via empirical colors, or more accurately, those corrected to a theoretical or an empirically determined stellar photosphere. To gain a complete picture of the disk-dissipation process, we want to do this over a range of wavelengths, ideally, and of course over a range of ages.

Independent of the wavelength studied or the mean age of the sample, there is a range of infrared excess properties observed among young pre-main sequence stars. For example, color excess distributions (e.g. 8-24 $\mu \mathrm{m}$ and 3.6-8 $\mu \mathrm{m}$ from Spitzer, or $\mathrm{K}-\mathrm{L}$ and $\mathrm{H}-\mathrm{K}$ from the ground) span a wide range of values at the youngest ages. Toward older ages, however, the distributions narrow, and eventually reflect just the error distribution. Hernandez et al (2007), for example, show the frequency distribution of near- to mid-infrared spectral-energy distribution slopes in several clusters $<5 \mathrm{Myr}$ of age. The distributions are double-peaked, indicative of the well-known bimodal behavior between 'disked' and 'non-disked' stars referred to earlier in the paper. Hillenbrand et al (2009) show similar near-infrared color excesses over photospheric values, which are broadly populated rather than double-peaked, and are indicative of the range in accretion properties exhibited by young stars. From overall consideration of star-forming regions having nominally different ages, a trend of decreasing dust disk (mean) frequency toward older (mean) ages is observed.

\subsection{Disk-dissipation trends with stellar radius}

Short infrared wavelengths trace hot dust, to a maximum temperature $\sim 1500-2000 \mathrm{~K}$. Near-infrared excesses (JHK bands) thus sample material at $\sim 0.03-0.1 \mathrm{AU}$, while mid-infrared excesses (LMNQ bands or Spitzer IRAC camera) typically $\sim 0.2-10 \mathrm{AU}$ and far infrared and sub-mm/mm wavelengths typically $\sim 10-100 \mathrm{AU}$. Stellar temperature/luminosity are also influential in setting these ranges; the above numbers are relevant for a young 'solar-type' star. It should not be forgotten that there are significant radiative transfer effects introduced on top of this simple picture due to radial and vertical disk structure as well as grain properties. Regardless, in the innermost disk regions, a lack of $1-2 \mu \mathrm{m}$ excess observable from the ground implies $<10^{-5} M_{\odot}$ in dust at the relevant temperature of $\sim 1000 \mathrm{~K}$. In the mid-disk range, lack of 8 and $24 \mu \mathrm{m}$ excess with Spitzer also implies $<1 M_{\text {Earth }}$ in $\sim 100 \mathrm{~K}$ dust. In the outer disk, where the prevalent upper limits to sub-mm and $\mathrm{mm}$ excesses are less restrictive, only $<10^{-4} M_{\odot}$ in $30-50 \mathrm{~K}$ dust is implied by non-detection.

In the near-infrared (measuring typically sub-micron grains), we believe that the excesses are tied directly to the accretion of material from the inner disk to the star. Indeed, the general decay of near-infrared excess with advancing stellar age over $1-10 \mathrm{Myr}$ is well reproduced by a similar decay in the frequency of $\mathrm{H} \alpha$ emission for the same stars (Dahm (2005), see also Mohanty et al (2005), Jayawardhana et al (2006)). These arguments are buttressed by older evidence in the literature for a $1: 1$ correlation among members of Taurus-Auriga between thermal emission from disks and gas emission from accretion (Edwards et al 1994). Characteristic timescales are a few Myr (see Hillenbrand (2005) for more in-depth discussions) and functional fits to the accretion diagnostics of the form time ${ }^{-1}$, time $^{-2}$ or $\mathrm{e}^{- \text {time }}$ may be appropriate.

In the mid-infrared (measuring typically micron-sized grains), evidence is rapidly building from Spitzer (Carpenter et al 2006, Dahm and Hillenbrand 2007, Lada et al 2006, Megeath et al 2005, Sicilia-Aguilar et al 2006, Silverstone et al 2006), but was also apparent from the ground (Mamajek et al 2004) that a similar though, perhaps, slightly longer decay time can be inferred. In the sub-mm and $\mathrm{mm}$ (measuring potentially large grains up to tens or hundreds of microns in size), no trends are available yet, but there is a clear cliff of detectability that appears intimately tied to disk presence at shorter wavelengths. The data are consistent with all CTTS having disks with mass $>10^{-4} M_{\odot}$ and $<10 \%$ of WTTS having substantial sub-mm disks (Andrews and Williams 2005).

There are, of course, some caveats to the above. One limititation is observational sensitivity, meaning that the weakest or lowest mass disks are not detectable. This is generally due either to raw observational sensitivity limits, or to lack of calibration precision which effectively destroys capability to detect excesses within that uncertainty level. A second limitation is in the physical interpretation of employed disk diagnostics, i.e. how well we understand the physics leading to excess flux and how we correlate wavelength of the excess with temperature/location of the emitting disk material. A final uncertainty, as discussed in detail above, relates to the accuracies of stellar ages.

How do we interpret the current trends in disk dissipation versus wavelength (sic, disk radius)? We still—and will for some time-lack an unambiguous mapping between observed spectral energy distributions and physical disks. However, coming back to the so-called 'transitional disks', it is notable that the observations indicate morphologies suggestive of the dust becoming optically thin first at shorter wavelengths and only later at longer wavelengths. This apparent ordering could be due to the processes associated with physical disk draining (e.g. via accretion onto the central star, through launching 


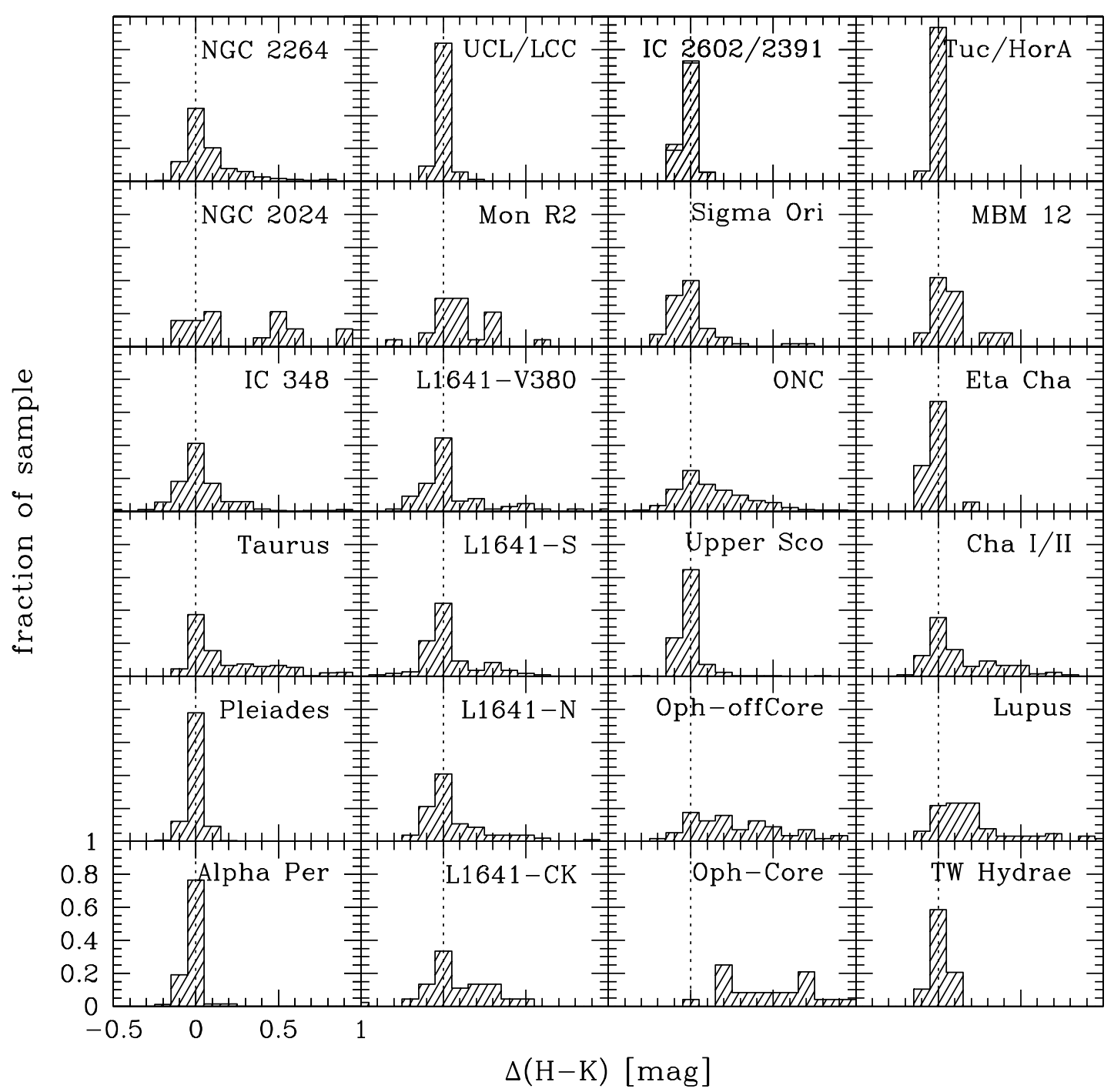

Figure 1. Normalized frequency distribution of near infrared excesses indicative of hot circumstellar dust associated with accretion processes in $<1-100$ Myr old stars. The younger clusters show a range of near-infrared excess amplitudes (including zero excess) while the older clusters are more strongly peaked at zero excess.

into a stellar wind or by photo-evaporative processes that perhaps progress from the inner disk to the outer disk) or to transformation of disk material (e.g. the growth of small interstellar-like particles into grains larger than $\lambda / 2 \pi$, which are then generally not detectable at wavelength $\lambda$ ).

Indeed, an open question is whether disk material dissipates at all radii simultaneously, or whether inner disks disappear first, as holes develop on a viscous evolution, photo-evaporative, or dynamical timescale and propagate outwards. The expected times are in all the cases comparable with the dynamical timescale - days in the inner disk and $\sim 10^{5}$ years in the outer disk. Although fast, these are perhaps not as fast enough as to produce the 'CTTS/WTTS switch' that is observed. One way of describing empirically the evolutionary process may be via measurement of the slope of the infrared excess as it departs the stellar photosphere, versus the wavelength at which the departure occurs (e.g. Cieza et al 2007). It is proposed that grain growth implies clearing over a large range of wavelengths near-simultaneously, while dynamical or photo-evaporative effects would proceed from the interior of the disk outward. It is suggested that these scenarios would have different tracks in such a diagram.

\subsection{Disk-dissipation trends with stellar mass}

Another very clear trend from Spitzer data is the mass dependence of circumstellar disk dissipation. Carpenter et al (2006) and Dahm and Hillenbrand (2007) show evidence in two different clusters of nominal age $5 \mathrm{Myr}$ that the disks remain around only the lowest mass (K and $\mathrm{M})$ stars. This is the first definitive evidence of the long-espoused notion that higher mass stars may lose their disks more rapidly. The finding is as one might infer from some combination of the larger radiation fields and the higher inferred mass accretion rates (Calvet et al 2005, Garcia Lopez et al 2006) for more massive stars, and is consistent with an interpretation that the trend is driven by initial conditions (Alexander and Armitage 2005). Further, the disks remaining in these two clusters (Upper Sco and NGC 2362), as well as others (such as $\lambda$ Ori, $\sigma$ Ori and Orion OB1) in even more recent literature, are weakened in strength or amplitude of the measured excess, relative to the larger excess values observed toward stars of similar mass in younger regions.

How do we interpret the current trends in disk dissipation versus stellar age as a function of stellar mass? Clearly, 
the fact that we can even use terminology such as 'disk fraction' suggests that at any given age some stars have disks while others do not. In other words, there is dispersion-indeed, diversity - in the timescale for disk dispersal. This is true even among objects with apparently identical properties otherwise (stellar mass, metallicity, star-formation environment). Further, the mass-dependence of both accretion properties and thermal dust-emission processes is clear, and also accompanied by dispersion. An open question is whether the observed trends should be interpreted as dispersion in initial disk properties, dispersion in the onset of some common disk evolutionary switch or dispersion among objects in the relative importance of the various possible disk-dissipation mechanisms.

\section{Quantifying diversity}

The wide dispersion in observed spectral-energy distributions (indicative to first order of dust disk geometry and, in detail, of radiative transfer effects) is especially prevalent at ages of <1-3 Myr. By $\sim 5$ Myr the diversity settles down, with observed disks predominantly 'weak' and by $\sim 10 \mathrm{Myr}$ most disks are undetectable or nonexistent, with only a very few stars having 'strong' disks. What we really want to know, however, is not the evolution of observational parameters - e.g. disk fraction, infrared excess amplitude, SED slope, $\lambda_{\text {onset }}$, optical veiling, emission line fluxes, etc - or even the evolution of the corresponding physical parameters, such as disk radial and vertical structure, total dust mass, grain size distribution, $\left(\mathrm{d} M_{\text {accretion }} / \mathrm{d} t\right)$, gas mass, etc. Rather, we aim to understand a higher level question: the frequency distribution of the lifetime of dust (as well as gaseous) material above a certain mass, as a function of disk radius. How many disks last only 0.1 Myr year, how many $1 \mathrm{Myr}$, how many 5, 10 and 20 Myr?

This is approached, in principle, via building the distribution of disk lifetimes as a function of wavelength. Ideally, we want to go even further and understand the mean and dispersion in the evolution of physical quantities, such as the disk surface density distribution, $\Sigma(r)$. Are such distributions Gaussian or do they exhibit long tails? Does planet formation occur throughout the distribution or only within the long tails? Is the circumstellar evolutionary process different for stars of different masses?

\subsection{Gas!}

We are unlikely to be able to discern in great detail what has happened to the ubiquitous early-stage circumstellar dust, via studies of the dust. Instead, we might turn to studies of gas. In the grain-growth scenario for planet formation, the gas is likely to remain and to be available for continued diskaccretion/outflow processes. Conversely, in a disk-clearing scenario accompanying planet formation, the gas likely disappears via the same or a similar mechanism to that causing the dust removal. As in so many other areas of disk evolution, the less observationally constrained gas is key to our astrophysical understanding. Some of these same points have been made recently by Najita et al (2007).
What of current gas constraints? Emission in $\mathrm{H}_{2}$ and $\mathrm{CO}$ has been detected from the ground (Bary et al 2002, 2003, Bitner et al 2007, Ramsay Howat and Greaves 2007, Salyk et al 2007, Weintraub et al 2000) and measures warm-to-hot gas in disk surface layers or inner disk regions. With Spitzer, [Ne II] at $12.8 \mu \mathrm{m}$ has been observed (Lahuis et al 2007, Pascucci et al 2007) and may actually measure the photo-evaporative flow (Herczeg et al 2007). Spitzer has also detected $\mathrm{OH}, \mathrm{H}_{2} \mathrm{O}$ and simple organic molecules in (Salyk et al 2008, Carr and Najita 2008). Gas studies, e.g. with the forthcoming Herschel, are needed. Noteworthy is that the ages inferred for several of the very few systems with measured gas content of any significance are relatively old - 5-10 Myr!

\section{Closing in on the future}

One point to emphasize, in particular, regarding circumstellar disk evolution is that whatever happens, happens fast. An increasingly important limit to our understanding is thus the large uncertainty in stellar ages that leads to large uncertainty in disk-evolution times, since $\Delta \tau_{\text {evolution }}<\delta \tau_{\text {age }}$. In other words, the phenomenon occurs on timescales comparable with or less than a stellar age resolution element. Not so much later in the disk evolution process, as primordial disks are dissipating, it is likely that debris disks are arising. For these gas-poor disks, in contrast to the gas-rich primordial disk situation, $\Delta \tau_{\text {evolution }}>\delta \tau_{\text {age }}$. In other words, the phenomenon occurs on much longer timescales than the uncertainties in stellar ages. Also needed for progress on disk evolution is semantic agreement on, and a common definition for 'time,' especially concerning a meaningful zero point.

Better understanding is needed of this transition from 'primordial' disks - in which the mean grain size is increasing with time as material agglomerates to form planets - and 'debris' disks - in which the mean grain size is decreasing with time as material is destroyed in the planet-induced stirring of planetesimals and resulting collisional cascade followed by radiative blowout or drag. Because the youngest examples of debris disks overlap in age with the oldest-known (accreting, even!) primordial disks, we are going to have to be somewhat careful in parsing the data in this age range (3-15 Myr). Vestiges of both types of disks are likely present.

As an example, Carpenter et al (2006) may be seeing in their $5 \mathrm{Myr}$ old sample evidence for debris disks surrounding some of the earlier type (A-F) stars and unevolved primordial disks surrounding the later type $(\mathrm{K}-\mathrm{M})$ stars. It has also been argued by Metchev et al (2005) in the case of the $\sim 12 \mathrm{Myr}$ old M-type star AU Mic, that while the inner regions of the spatially resolved disk are collisionally evolved debris, the outer regions are most likely pristine material that is still part of the remnant primordial disk. The formation of debris disks, commonly accepted as evidence of formed planetesimals, can easily be confused with long-lived primordial disks. Extreme caution and probably cleverness in the interpretation of observational data is needed. Gas studies are extremely promising in this regard.

Finally, we note that the relation between processes of disk evolution and those of planet formation is increasingly clear. We are currently in a stage of great luxury in being able to refine, based on the substantial progress over the past 
several years, the questions we can afford to ask of theory and of obtained/future data. There is a developing need to match the increasingly predictive theory with increasingly detailed observations.

\section{References}

Adams F C et al 2008 Phys. Scr. T130 014029

Aikawa Y and Nomura H 2008 Phys. Scr. T130 014011

Alexander R D and Armitage P J 2005 Astrophys. J. Lett. 63983

Alexander R D and Armitage P J 2007 Mon. Not. R. Astron. Soc. 375500

Andrews S M and Williams J P 2005 Astrophys. J. 6311134

Andrews S M and Williams J P 2007 Astrophys. J. 6711800

Bary J S, Weintraub D A and Kastner J H 2002 Astrophys. J. Lett. 57673

Bary J S, Weintraub D A and Kastner J H 2003 Astrophys. J. 586 1136

Bauer F, Dullemond C P and Henning Th 2008 Astron. Astrophys. 480859

Bertout C, Siess L and Cabrit S 2007 Astron. Astrophys. J 47321

Bitner M A, Richter M J, Lacy J H, Greathouse T K, Jaffe D T and Blake G A 2007 Astrophys. J. Lett. 66169

Calvet N, Briceno C, Hernandez J, Hoyer S, Hartmann L, Sicilia-Aguilar A, Megeath S T and D'Alessio P 2005 Astron. J. 129935

Carpenter J M, Mamajek E E, Hillenbrand L A and Meyer M R 2006 Astrophys. J. Lett. 65149

Carr J and Najita J R 2008 Science 3191504

Cieza L et al 2007 Astrophys. J. 667308

Dahm S E 2005 PhD Thesis University of Hawaii

Dahm S E and Hillenbrand L A 2007 Astron. J. 1332072

Draine et al 2006 Astrophys. J. $\mathbf{6 3 6} 1114$

Dullemond C P et al 2008 Phys. Scr. T130 014015

Dutrey A, Guilloteau S, Duvert G, Prato L, Simon M, Schuster K and Menard F 1996 Astron. Astrophys. 309493

Edwards S et al 1994 Astron. J. 1081056

Foing B H et al 2008 Phys. Scr. T130 014026

Furlan E et al 2006 Astrophys. J. Suppl. 165568

Garcia Lopez R, Natta A, Testi L and Habart E 2006 Astron. Astrophys. 459797

Gorti U and Hollenbach D 2008 arXiv 0804.3381

Gullbring E, Hartmann L, Briceno C and Calvet N 1998 Astrophys. J. 492323

Hartigan P, Edwards S and Ghandour L 1995 Astrophys. J. 452736

Hartmann L, Calvet N, Gullbring E and D'Alessio P 1998 Astrophys. J. 495385

Hartmann L et al 2005 Astrophys. J. 629881

Henning Th 2008 Phys. Scr. T130 014019

Herczeg G J, Najita J, Hillenbrand L A and Pascucci I 2007670504

Hernandez J et al 2007 Astrophys. J. 66621067

Hillenbrand L A 2005 review article to appear in 'A Decade of Discovery: Planets Around Other Stars' (STSCI Symposium Series vol. 19) ed M Livio Preprint astro-ph/0511083

Hillenbrand L A, Bauermeister A and White R J 2007 ASP Conf. Ser. 384200

Hillenbrand L A, Bauermeister A and White R J 2008 in preparation
Hillenbrand L A, Meyer M R and Carpenter J M 2009 in preparation Hillenbrand L A and White R J 2004 Astrophys. J. 604741

Hirota T et al 2007 PASJ 6037

Hollenbach D, Johnstone D, Lizano S and Shu F 1994 Astrophys. J. 428654

Jayawardhana R, Coffey J, Scholz A, Brandeker A and van Kerkwijk M H 2006 Astrophys. J. 6481206

Jeffries R D 2007 Mon. Not. R. Astron. Soc. 3761109

Lada C J et al 2006 Astron. J. 1311574

Lahuis F, van Dishoeck E F, Blake G A, Evans N J II, Kessler-Silacci J.E and Pontoppidan K M 2007 Astrophys. $J$. 665492

Low F J, Smith P S, Werner M, Chen C, Krause V, Jura M and Hines D C 2005 Astrophys. J. 6311170

Mamajek E E, Meyer M R, Hinz P M, Hoffmann W F, Cohen M and Hora J L 2004 Astrophys. J. 612496

Megeath S T, Hartmann L, Luhman K L and Fazio G G 2005 Astrophys. J. Lett. 634113

Menten K M, Reid M J, Forbrich J and Brunthaler A 2008 Astron. Astrophys. 474515

Metchev S A, Eisner J A, Hillenbrand L A and Wolf S 2005 Astrophys. J. 622451

Mohanty S, Jayawardhana R and Basri G 2005 Astrophys. J. 626 498

Monin J-L, Clarke C J, Prato L and McCabe C 2007 Protostars and Planets $V$ ed B Reipurth, D Jewitt and K Keil (Tucson: University of Arizona Press) p 395

Muzerolle J, Hillenbrand L, Calvet N, Briceno C and Hartmann L 2003 Astrophys. J. 592266

Najita J R, Strom S E and Muzerolle J 2007 Mon. Not. R. Astron. Soc. 378369

Natta A 2008 Phys. Scr. T130 014014

Nordh L et al 1996 Astron. Astrophys. Lett. 315185

Osterloh M and Beckwith S V W 1995 Astrophys. J. 439288

Pascucci I et al 2007 Astrophys. J. 663383

Pudritz R E, Ouyed R, Fendt Ch and Brandenburg A 2007 Protostars and Planets $V$ ed B Reipurth, D Jewitt and K Keil (Tucson: University of Arizona Press) p 277

Ramsay Howat S K and Greaves J S 2007 Mon. Not. R. Astron. Soc. 3791658

Salyk C, Blake G A, Boogert A C C and Brown J M 2007 Astrophys. J. Lett. $\mathbf{6 5 5} 105$

Salyk C, Pontoppidan K M, Blake G A, Lahuis F, van Dishoeck E F and Evans II N J 2008 Astrophys. J. Lett. 67649

Sandstrom K M et al 2007 Astrophys. J. 6671161

Sicilia-Aguilar A, Hartmann L, Furesz G, Henning T, Dullemond C and Brandner W 2006a Astron. J. 1322135

Sicilia-Aguilar A et al 2006b Astrophys. J. 638897

Silverstone M D et al 2006 Astrophys. J. 6391138

Simon M and Prato L 1995 Astrophys. J. 450824

Skrutskie M F, Dutkevitch D, Strom S E, Edwards S, Strom K M and Shure M A 1990 Astron. J. 991187

Takeuchi T, Clarke C J and Lin D N C 2005 Astrophys. J. 627286

Tout C A, Livio M and Bonnell I A 1999 Mon. Not. R. Astron. Soc. 310360

Watson D M et al 2007 Preprint $/ 0704.1518$

Weintraub D A, Kastner J H and Bary J S 2000 Astrophys. J. 541 767

White R J and Basri G 2003 Astrophys. J. 5821109

Wolk S J and Walter F M 1996 Astron. J. 1112066 